\title{
LETTER TO THE EDITOR Engineered trimeric ACE2 binds viral spike protein and locks it in "Three-up" conformation to potently inhibit SARS-CoV-2 infection
}

\author{
Cell Research (2021) 31:98-100; https://doi.org/10.1038/s41422-020-00438-w
}

\section{Dear Editor,}

Coronavirus disease 2019 (COVID-19) caused by severe acute respiratory syndrome coronavirus 2 (SARS-CoV-2) has resulted in a severe global pandemic. Following SARS-CoV, SARS-CoV-2 is yet another emergent beta-coronavirus threatening human health. ${ }^{1}$ However, seventeen years after the SARS pandemic, no targeted vaccines or therapeutics have been approved for SARS, while some of them might have held promise for treating COVID-19. Many neutralizing antibodies against SARS-CoV-2 are currently being developed. However, RNA viruses are known to have high mutation rates. Many SARS-CoV-2 mutations have already been identified such as $\mathrm{D} 614 \mathrm{G}^{2}$ and these resultant mutation strains might escape the effects of the current SARS-CoV-2 neutralizing antibodies. The appearance of COVID-19 after SARS indicates the likely emergence of other coronavirus pandemics in the future. Thus, therapeutics broadly effective against SARS-CoV-2 and mutants, even other SARSCoV-2-related coronaviruses (SARSr-CoVs), are highly desirable. Both SARS-CoV-2 and SARS-CoV bind ACE2 for cell entry, suggesting a general use of ACE2 by SARS-CoV-2 mutants and future related coronaviruses. Therefore, proteins engineered based on wild-type ACE2 might exhibit the best broad neutralizing activity and avoid the mutational escape.

Coronavirus infection can cause shedding of ACE2 resulting in a decreased level of ACE2 expression. ${ }^{3,4}$ This phenomenon is closely related to acute lung injury, and replenishing soluble ACE2 could alleviate acute respiratory distress syndrome (ARDS). ${ }^{3,4}$ It has been shown that recombinant soluble ACE2 could inhibit SARS-CoVs infection in cells and organoids. ${ }^{5,6}$ One clinical trial (NCT04335136) was also registered to use recombinant ACE2 to treat COVID-19. However, recombinant soluble ACE2 inhibits viral infection at relatively high concentrations, ${ }^{5,6}$ therefore, it may not be an optimal inhibitor. Since spike proteins of SARS-CoVs function as trimers, ${ }^{7}$ we reasoned that an engineered trimeric ACE2 protein could potentially bind up to three receptor binding domains (RBD) on the spike protein, which would dramatically increase binding affinity and thus potently inhibit SARS-CoVs (Supplementary information, Fig. S1).

To develop such trimeric ACE2 proteins, we chose a C-terminal domain of T4 fibritin (foldon), ${ }^{8}$ or a three helix bundle (3HB). ${ }^{9}$ We also analyzed the reported SARS-CoVs spike protein structures and estimated that distances between RBDs on the same spike protein could range from 60 to $100 \AA$ when these three RBDs are in the three up conformation. ${ }^{7,10}$ We then chose a flexible (GGGGS) ${ }_{5}$ linker, or a more rigid $(E A A A K)_{5}$ linker, to construct four trimeric ACE2 proteins: ACE2-flexible-3HB, ACE2-rigid-3HB, ACE2-flexible-foldon and ACE2-rigid-foldon. ${ }^{11}$ In addition, we constructed two trimeric ACE2 proteins with a short linker GGGS (ACE2-short-3HB, ACE2short-foldon) and a monomeric ACE2 as control (Supplementary information, Fig. S2).

Binding affinities between ACE2 proteins and the prefusion stabilized trimeric SARS-CoV-2 spike protein ectodomain (S-ECD) were determined by ELISA (Supplementary information, Fig. S3). ${ }^{7}$ ACE2 monomer binds S-ECD with $\mathrm{IC}_{50}$ of $27 \mathrm{nM}$. Significant enhancement of the binding affinity was observed for trimeric ACE2 proteins, and the rigid linker constructs showed the highest binding affinities, including ACE2-rigid-3HB and ACE2rigid-foldon, which both bound S-ECD with $\mathrm{IC}_{50}$ of $30 \mathrm{pM}$. Trimeric ACE2 proteins with short linkers had lower binding affinities (Supplementary information, Fig. S3).

Biolayer interferometry was used to further analyze the binding affinity between ACE2 proteins and S-ECD (Fig. 1a-e). Trimeric ACE2 proteins again exhibited dramatically increased binding affinities. $K_{D}$ for ACE2-flexible-3HB/S-ECD was $4.4 \mathrm{nM}$ while $K_{D}$ for ACE2-flexiblefoldon/S-ECD went down to $0.34 \mathrm{nM}$. Both $A C E 2-$ rigid-3HB and $A C E 2$-rigid-foldon bound S-ECD extremely tight, with $\mathrm{K}_{\mathrm{D}}<1 \mathrm{pM}$. Further decreasing loading of S-ECD on streptavidin sensors did not affect ACE2 proteins binding, suggesting intramolecular avidity binding between trimeric ACE2s and S-ECD (Supplementary information, Fig. S4).

Next, we assessed the inhibitory activities of these trimeric ACE2 decoy proteins using SARS-CoV-2 and SARS-CoV pseudotyped viruses (Fig. 1f, g). ACE2 monomer can only inhibit SARS-CoV-2 pseudotyped virus at high concentration with $I_{50}>50 \mathrm{nM}$. As expected, trimeric ACE2 with flexible linkers showed much better inhibitory activities. ACE2-flexible-3HB inhibited SARS-CoV-2 infection with $\mathrm{IC}_{50}$ of $3.46 \mathrm{nM}$, while ACE2-flexible-foldon had better inhibitory activity with $\mathrm{IC}_{50}$ of $1.58 \mathrm{nM}$. Rigid-linker trimeric ACE2 proteins again displayed the highest inhibitory activities. ACE2-rigid$3 \mathrm{HB}$ and $\mathrm{ACE} 2$-rigid-foldon showed similar $\mathrm{IC}_{50}$ of 0.40 and $0.48 \mathrm{nM}$, respectively. Short-linker trimeric ACE2 proteins did not show potent inhibitory activities (Supplementary information, Fig. S5). Similar results were observed with SARS-CoV pseudotyped virus inhibition, and ACE2-rigid-foldon had the best inhibitory activity with $\mathrm{IC}_{50}$ of $2.41 \mathrm{nM}$. Thus, the ACE2-rigid-foldon construct is the most potent trimeric ACE2, which is designated T-ACE2 hereafter.

We then asked whether T-ACE2 could also inhibit SARS-CoV-2 mutants and SARSr-CoVs. We tested T-ACE2 inhibitory activities on eight naturally occurring SARS-CoV-2 mutants, including seven RBD domain mutants ${ }^{12}$ and D614G mutant, and two SARSr-CoVs (WIV1 and Rs3367). We found that T-ACE2 could potently inhibit all these viruses at low concentrations ( $\mathrm{nM}$ to sub-nM IC 50 , Fig. 1h; Supplementary information, Fig. S6). The observed inhibitory activities prompt us to speculate that T-ACE2 might inhibit more SARS-CoV-2 novel mutants. We further tested the inhibition of authentic SARS-CoV-2 infection by T-ACE2 (Fig. 1i, j). Importantly, we found that T-ACE2 could also potently inhibit SARS-CoV-2 infection with $\mathrm{IC}_{50}$ of $1.88 \mathrm{nM}$, which is consistent with our binding affinity and pseudotyped virus inhibition results.

We hypothesized that properly designed trimeric ACE2 might engage more than one RBDs from the trimeric spike protein and thus dramatically increase binding affinity through avidity effect. 

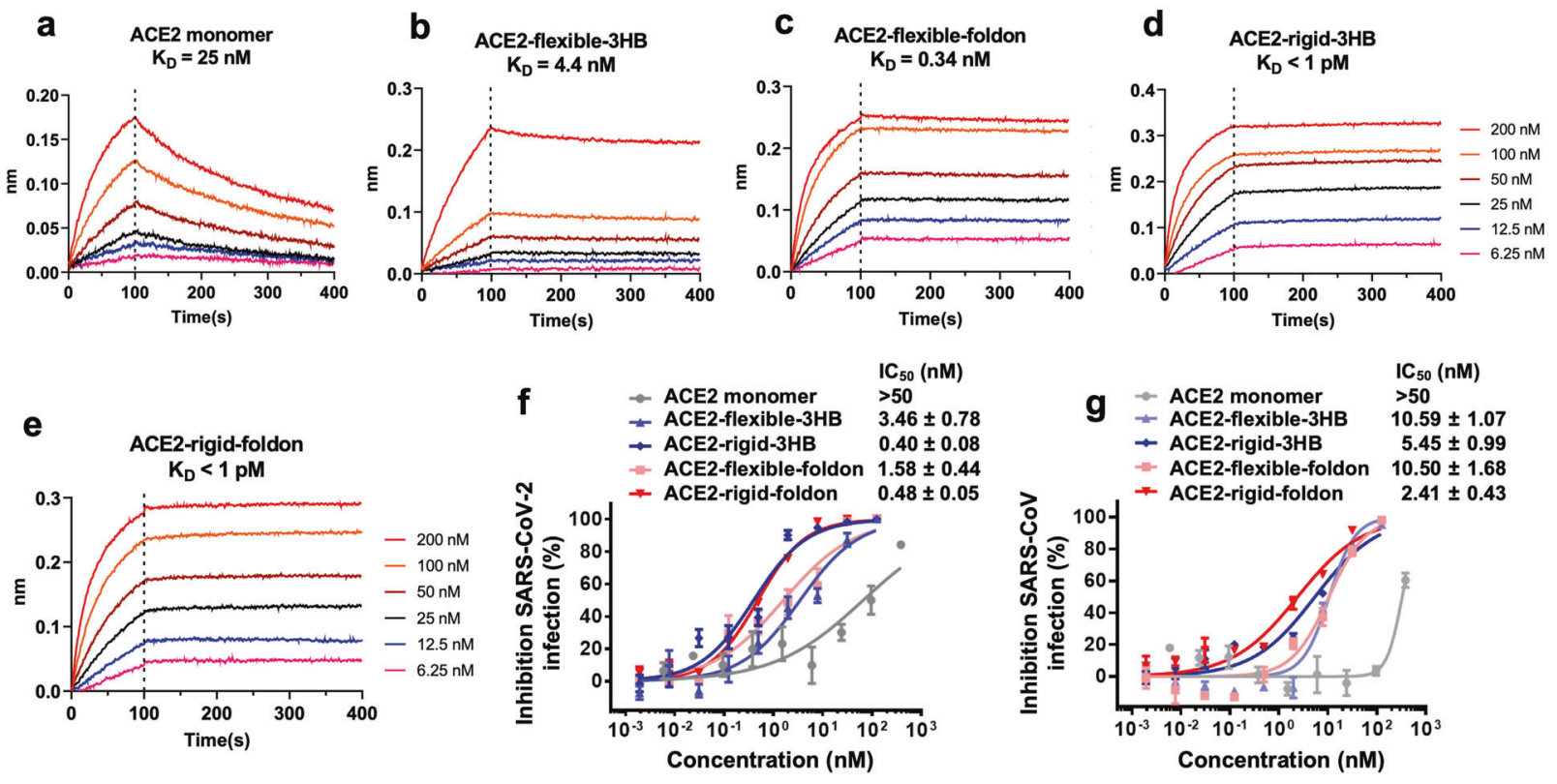

\begin{tabular}{|c|c|c|c|c|c|c|c|c|c|c|}
\hline \multirow{2}{*}{ Virus } & \multicolumn{8}{|c|}{ SARS-CoV-2 mutant } & \multicolumn{2}{|c|}{ SARSr-CoV } \\
\hline & V341I & F342L & V367F & R408I & A435S & G476S & V483A & D614G & WIV1 & Rs3367 \\
\hline $\begin{array}{l}\text { Inhibition } \\
\left(\mathrm{IC}_{50}, \mathrm{nM}\right)\end{array}$ & 1.44 & 2.83 & 0.16 & 0.57 & 0.79 & 1.15 & 1.18 & 0.61 & 0.1 & 1.51 \\
\hline
\end{tabular}
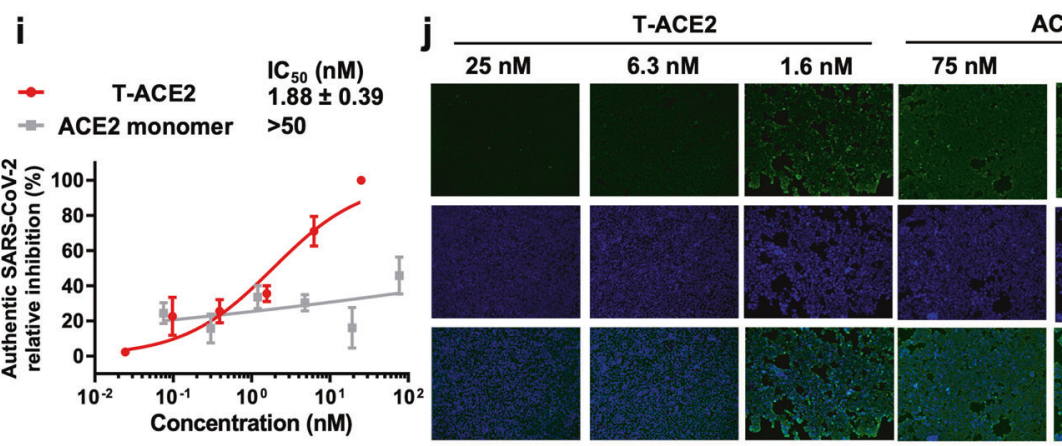

ACE2 monomer

Virus control
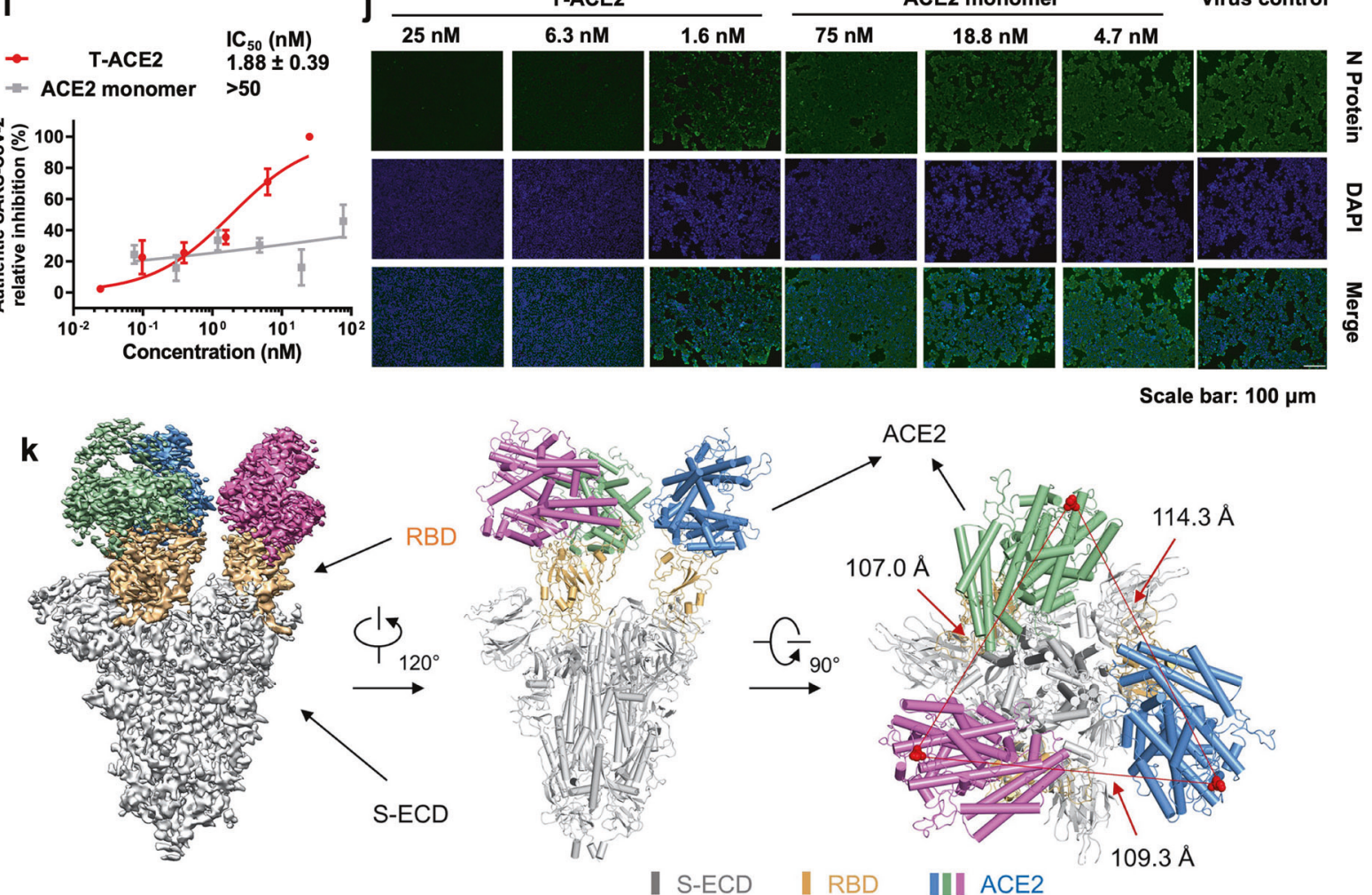

Scale bar: $100 \mu \mathrm{m}$

\section{|| S-ECD}

\section{RBD}

Fig. 1 ACE2 proteins binding affinities, virus inhibitory activities and T-ACE2/S-ECD complex structure. a-e Binding affinities measured using biolayer interferometry between ACE2 proteins and S-ECD. $\mathbf{f}$ ACE2 proteins inhibition of SARS-CoV-2 pseudotyped virus. $\mathbf{g}$ ACE2 proteins inhibition of SARS-CoV pseudotyped virus. h ACE2-rigid-foldon (T-ACE2) inhibition of SARS-CoV-2 mutants and SARSr-CoVs pseudotyped viruses. i ACE2-rigid-foldon (T-ACE2) inhibition of SARS-CoV-2 virus. Vero E6 cells were infected with authentic SARS-CoV-2 $(\mathrm{MOI}=0.0033)$, and inhibitory effects were evaluated using quantitative real-time PCR (qPCR). $\mathbf{j}$ Immunofluorescence microscopy of SARSCoV-2 infection upon treatment of T-ACE2 or ACE2 monomer. $\mathbf{k}$ Cryo-EM structure of the T-ACE2/S-ECD complex. The domain-colored cryoEM map of the complex is shown on the left. Two perpendicular views of the overall structure are shown on the right. The three ACE2 monomers from T-ACE2 are colored blue, green and violet, respectively. The RBDs of the S-ECD are colored orange. Distances between each ACE2 C-terminus within T-ACE2 are colored red. 
To further validate this hypothesis, we determined the T-ACE2/SECD complex structure using cryo-electron microscopy (cryo-EM) (Supplementary information, Figs. S7-S9 and Table S1). Strikingly, in the complex, the spike protein adopts only one conformation: the "three-up" RBD conformation. The complex has a nearly perfect three-fold symmetry. Most importantly, all three RBDs bind to three ACE2s simultaneously (Fig. 1k). The binding interactions between ACE2 and RBD essentially agree with previous studies, and the three copies from the complex are well aligned (Supplementary information, Figs. S9, S10). ${ }^{13}$ But our spike protein conformation is very different from the previously reported prefusion stabilized spike protein structures where only one or no RBD is in the up position. Recent complex structures between SARS-CoV-2 spike protein and ACE2 monomer indicate that monomer ACE2 binding can induce conformational changes of spike protein and that some spike proteins can have two or three RBDs in the up position to bind up to three ACE2s. ${ }^{14}$ In our structure, the unique "three-up" RBD conformation in all the spike proteins should have been induced by our T-ACE2.

Whether this T-ACE2-induced spike protein conformation change represents a transition state during virus infection needs to be further elucidated. Full-length ACE2 protein functions as a dimer, ${ }^{13}$ the two monomers from this ACE2 dimer are related by two-fold symmetry, which are situated close in space, with the distance between each D615 being about $53 \AA$ A. Thus, the native dimeric ACE2 is unlikely to engage more than one RBD from the same spike protein without substantial conformational changes. It is however possible that ACE2 dimers on the cell surface might further cluster to induce more RBDs to adopt up conformation and help virus to transit from the prefusion state to the postfusion state.

In summary, we engineered trimeric ACE2 proteins based on wild-type ACE2 and showed that T-ACE2 could bind spike protein with extremely high affinity to potently inhibit all tested pseudotyped viruses including SARS-CoV-2, SARS-CoV, eight naturally occurring SARS-CoV-2 mutants, two SARSr-CoVs as well as authentic SARS-CoV-2. Carrying these advancements a few steps beyond, the modular design of T-ACE2 demonstrates that other oligomerization motifs and linkers could be further explored to improve the properties of T-ACE2 or higher oligomeric ACE2s. We believe that T-ACE2 represents a promising class of proteins to broadly inhibit SARS-CoVs and to treat virus-infected patients. Finally, the high binding affinity between T-ACE2 and spike protein suggests that T-ACE2 could also be useful for virus detection. The fact that T-ACE2 was engineered based on native ACE2 sequence renders such detection methods potential application for all SARS-CoVs and related viruses.

\section{ACKNOWLEDGEMENTS}

We thank the Cryo-EM facility, Supercomputer Center of Westlake University. We thank the Core Facility of Microbiology and Parasitology (SHMC) and the Biosafety Level 3 Laboratory at Shanghai Medical College of Fudan University, especially Di Qu, Yutang Li, Qian Wang, Zhiping Sun and Chengjian Gu for continuous support. We thank Tian Li, Xingyue Bao, Mohamad Sawan, Dan Ma and Huaizong Shen for helping protein and cell preparations. We thank Peihui Wang for sharing ACE2 plasmid. This work was supported by Westlake Education Foundation and Tencent Foundation, the National Megaprojects of China for Major Infectious Diseases (2018ZX10301403 to L.L.), the Natural Science Foundation of China (22077104, 32022037, 31971123, 81920108015, and 31930059), the Key R\&D Program of Zhejiang Province (2020C04001), and the SARS-CoV-2 emergency project of the Science and Technology Department of Zhejiang Province (2020C03129), and the Leading Innovative and Entrepreneur Team Introduction Program of Hangzhou.

\section{AUTHOR CONTRIBUTIONS}

B.D. conceived the project. L.G., K.Z. and M.Z. performed the biochemical experiments, W.B., X.W., W.X, X.C., and Y.L. performed the viral experiments. R.Y., Y.Z. and Y.L. solved the cryo-EM structure. B.D., L.L., Q.Z., Y.X. and S.J. designed and supervised the experiments, interpreted the data, wrote and revised the manuscript.

\section{ADDITIONAL INFORMATION}

Supplementary information accompanies this paper at https://doi.org/10.1038/ s41422-020-00438-w.

Competing interests: B.D., L.G. and W.B. are the inventors on a provisional patent filed by the Westlake University. The other authors declare no competing interests.

Liang Guo ${ }^{1,2,3}$, Wenwen $\mathrm{Bi}^{1,2,3}$, Xinling Wang ${ }^{4}$, Wei $\mathrm{Xu}^{4}$, Renhong Yan ${ }^{1,2,3}$, Yuanyuan Zhang ${ }^{1,2,3}$, Kai Zhao ${ }^{1,2,3}$, Yaning $\mathrm{Li}^{1,2,3^{\prime}}$, Mingfeng Zhang ${ }^{1,2,3}, \mathrm{Xia} \mathrm{Cai}^{4}$, Shibo Jiang ${ }^{4}{ }^{4}$, Youhua Xie $^{4}$, Qiang Zhou ${ }^{1,2,3}$, Lu Lu (iD) and Bobo Dang (D) ${ }^{1,2,3}$

${ }^{1}$ Key Laboratory of Structural Biology of Zhejiang Province, School of Life Sciences, Westlake University, Hangzhou, Zhejiang 310024,

China; ${ }^{2}$ Center for Infectious Disease Research, Westlake Laboratory of Life Sciences and Biomedicine, Hangzhou, Zhejiang 310024, China; ${ }^{3}$ Institute of Biology, Westlake Institute for Advanced Study,

Hangzhou, Zhejiang 310024, China and ${ }^{4}$ Key Laboratory of Medical Molecular Virology (MOE/NHC/CAMS), School of Basic Medical Sciences and Biosafety Level 3 Laboratory, Fudan University, Shanghai, 200032, China

These authors contributed equally: Liang Guo, Wenwen Bi, Xinling Wang, Wei Xu, Renhong Yan, Yuanyuan Zhang

These authors jointly supervised this work: Youhua Xie, Qiang Zhou, Lu Lu, Bobo Dang

Correspondence: Qiang Zhou (zhouqiang@westlake.edu.cn) or LuLu (lul@fudan.edu.cn) or Bobo Dang (dangbobo@westlake.edu.cn)

\section{REFERENCES}

1. Zhou, P. et al. Nature 579, 270-273 (2020).

2. Korber, B. et al. Cell 182, 812-827 (2020).

3. Imai, Y. et al. Nature 436, 112-116 (2005).

4. Kuba, K. et al. Nat. Med. 11, 875-879 (2005).

5. Hofmann, H. et al. Biochem. Biophys. Res. Commun. 319, 1216-1221 (2004).

6. Monteil, V. et al. Cell 181, 905-913 (2020).

7. Wrapp, D. et al. Science 367, 1260-1263 (2020).

8. Yang, X. et al. J. Virol. 76, 4634-4642 (2002).

9. Fletcher, J. M. et al. ACS Synth. Biol. 1, 240-250 (2012).

10. Kirchdoerfer, R. N. et al. Sci. Rep. 8, 15701 (2018).

11. Chen, X., Zaro, J. L. \& Shen, W. C. Adv. Drug Deliv. Rev. 65, 1357-1369 (2013).

12. Li, Q. et al. Cell 182, 1284-1294 (2020).

13. Yan, R. et al. Science 367, 1444-1448 (2020).

14. Zhou, T. et al. bioRxiv https://doi.org/10.1101/2020.07.04.187989 (2020). 